\title{
CONTROLLED DROPLET COALESCENCE IN AIR AND ITS APPLICATION TO MICROMIXING
}

\author{
Chuang-Yuan Lee, Hongyu Yu, and Eun Sok Kim \\ Department of Electrical Engineering-Electrophysics, \\ University of Southern California, Los Angeles, CA 90089-0271, USA
}

\begin{abstract}
This paper describes the controlled coalescence of liquid droplets in air by acoustic directional ejections and its application to micromixing. We intentionally created an asymmetrical electric field within the piezoelectric transducer to produce lopsided acoustic waves, which were focused on the liquid surface to obliquely eject nanoliter droplets. Effective micromixing was carried out in air through the continuous flight and rotations $(16,000 \mathrm{rad} / \mathrm{s})$ of the coalesced droplet. This digital fluid manipulation by directional droplet ejections allows complex analyses at high throughput and is practicable to versatile lifescience applications.
\end{abstract}

\section{INTRODUCTION}

The interest in on-chip biochemical analysis systems is growing rapidly, because they offer a fast, efficient and low cost assay. Rapid and homogenous mixing of two or more fluidic species is essential for these micro total analysis systems ( $\mu \mathrm{TAS}$ ). However, in a conventional microfluidic system, laminar flow occurs due to a low Reynolds number. Diffusion, dominant at the microscale, can take a relatively long time and decrease the overall system efficiency. In order to enhance the mixing performance, several passive micromixing methods have been developed by inducing chaotic advection and creating laminar shear, which require relatively long channels to lead the flow to a chaotic state [1].

Here we report the coalescence of liquid droplets in air resulting from acoustic directional ejections and its application to active mixing. This digital fluid manipulation by directional droplet ejections will be useful not only for micromixing but also for deoxyribonucleic acid and protein microarrays and other microfluidic applications.

\section{DESIGN AND FABRICATION}

A single directional ejector consists of an acoustic transducer and a lens [2]. By focusing acoustic energy on the liquid surface, acoustic radiation pressure acts to overcome the restraining force of surface tension and expels the droplets from an open space without any nozzle. To generate directional ejections, an asymmetrical electric field was intentionally created within the piezoelectric layer to produce lopsided acoustic waves (Fig. 1). A lead zirconate titanate (PZT) transducer with both top and bottom electrodes patterned into a sector shape was used in our design. Acoustic waves were only produced from the regions covered with patterned electrodes, resulting in uneven acoustic pressure distributions on the liquid surface.

Among the various patterns investigated, it was found that sectors with larger angles would incur lesser tilting for directional ejections, while smaller angles would suffer from severe electric fringing fields and consequent unstable ejections. There existed a tradeoff between directionality and stability, and a $90^{\circ}$ sector was observed to be the optimal design. Four ejectors were coordinately arrayed on a single chip to target one spot in the center with multiple liquids.

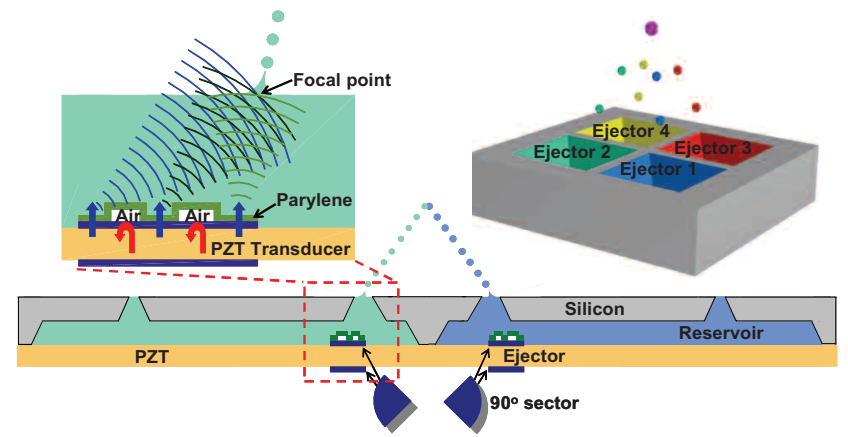

Figure 1. Schematic diagram of directional acoustic ejector array.

To fabricate the device, we first sectored top and bottom nickel electrodes on a $127-\mu \mathrm{m}$-thick PZT. Parylene was then deposited and patterned as the lens structure with photoresist as the sacrificial layer. After release, we adhesively bonded the PZT substrate to silicon wafers on which the $800-\mu$ m-deep (matching the lens focal length) reservoirs were microfabricated. Fig. 2 shows the photos of the fabricated PZT ejectors and silicon chambers before they were adhesively bonded together, and Fig. 3 shows the finished device in a dual-in-line package. (a)

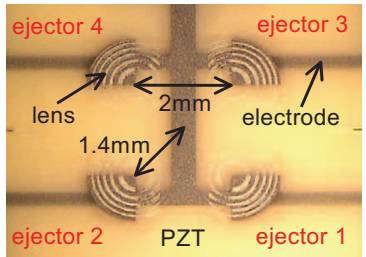

(b)

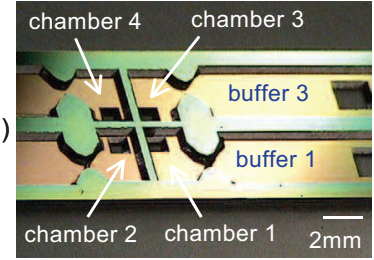

Figure 2. Photos of (a) ejector array and (b) chamber array.

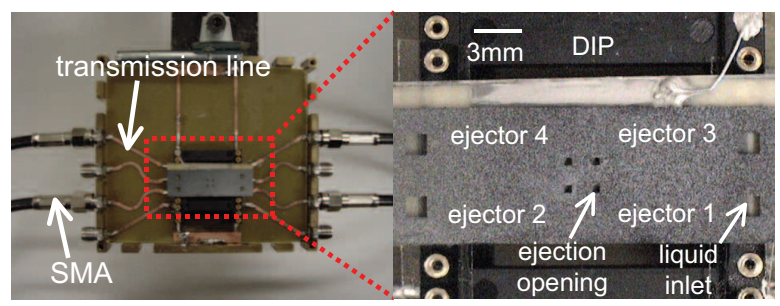

Figure 3. Fabricated device in a DIP package connected to transmission lines and SMA connectors.

\section{RESULTS AND DISCUSSION}

We first tested the performance of two ejectors (Ejectors 1 and 2) using water as the medium (Fig. 4). Two droplets $(80 \mu \mathrm{m}$ in diameter) broke off simultaneously from their own bulk liquids $200 \mu$ s after the rising edge of an applied pulse. They then approached each other at a speed of $2.3 \mathrm{~m} / \mathrm{s}$. At around $1100 \mu \mathrm{s}$, they arrived at the same place in air at the same time and coalesced into one larger droplet. The coalesced droplet kept on traveling with no velocity along the $x$ direction. Although the droplet size $(80 \mu \mathrm{m})$ is much smaller than the distance between the two ejectors 
(2 $\mathrm{mm})$, the two droplets still meet in a three-dimensional space because of the same ejection speed and precise directionality.

(a)

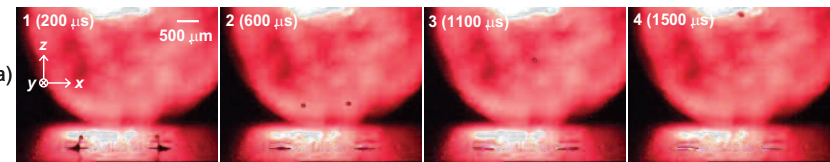

(b)

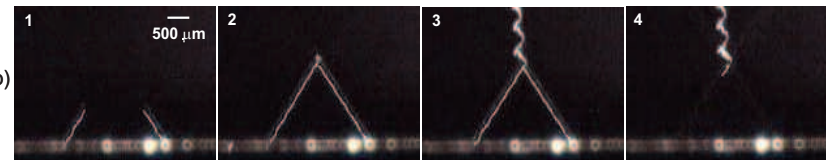

Figure 4. Droplet ejection process captured (a) with and (b) without strobe (Ejectors 1 and 2 activated). Ejectors were driven with \pm 80 - $V_{\text {peak-to-peak }}$ pulses of 16- $\mu$ s pulsewidth with $60-H z$ pulse repetition frequency $(P R F)$.

The ejection sequences of Ejectors 2 and 4 show the coalescence from another angle (Fig. 5). Coalescence was achieved by the sufficient collision kinetic energy to expel the air layer between the two colliding surfaces. The coalesced droplet continued to travel in air with the following characteristics. First, its gross traveling trajectory is in the additive direction of its composing droplets. Because the two composing droplets have the same density and size, this phenomenon is a consequence of the conservation of momentum. Second, as its airborne travel continues, the coalesced droplet rotates repeatedly with a periodicity of $400 \mu \mathrm{s}$ and a rotation velocity of $16,000 \mathrm{rad} / \mathrm{s}$.

(a)

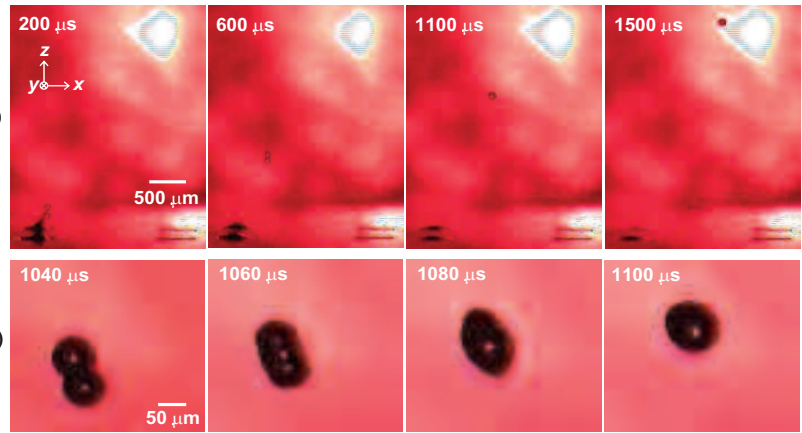

(c)

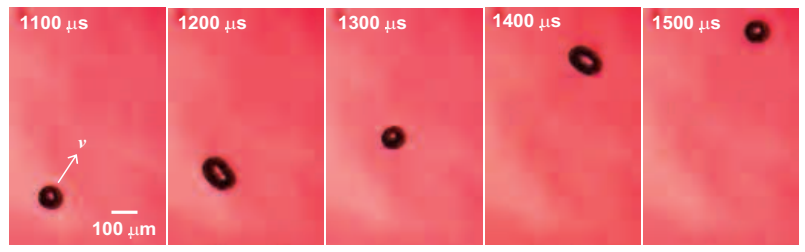

Figure 5. Droplets ejection process (Ejectors 2 and 4 activated). (a) Overview pictures of the ejection process. (b) Detailed droplets coalescence sequence around $1100 \mu \mathrm{s}$. (c) Rotations of the traveling coalesced droplet with a periodicity of $400 \mu \mathrm{s}$.

To demonstrate that more than two droplets can be merged in air, we actuated the four ejectors on a chip. The four droplets were created simultaneously, moved toward the center along the matched directions, and joined together in air to form a single coalesced droplet (Fig. 6).
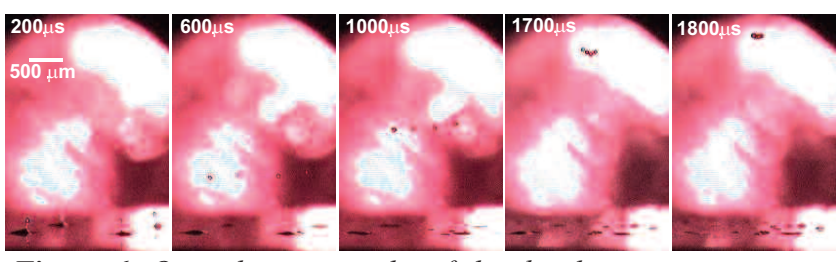

Figure 6. Optical micrographs of the droplets ejection process (all four ejectors activated). Ejectors were driven with $\pm 80-V_{\text {peak- }}$ to-peak pulses of 30- $\mu$ s pulsewidth with 60-Hz PRF.
The rotating characteristics of the coalesced droplet can be applied to micromixing for the following benefits. First, by mixing reagents in air without long channels on a substrate, the lab-on-achip real estate is greatly reduced. Second, through the active rotations after the coalescence, mixing is achieved more efficiently than by passive diffusion. To illustrate the mixing effect, two different mediums (water and red ink) were ejected by two separate directional ejectors. Because it was relatively difficult to perform mixing analysis for airborne droplets from the side-view micrographs, we examined the droplets collected on a glass slide placed 2-cm above the device (Fig. 7). With a rotation velocity of $16,000 \mathrm{rad} / \mathrm{s}$, the airborne droplet (containing the two liquids after the coalescence) was vigorously mingled through rotations of about 30 rounds before it was collected on the glass slide. The droplet homogeneity exhibited the excellent mixing by the precise directional ejections and the continuous flight and rotations after the coalescence.

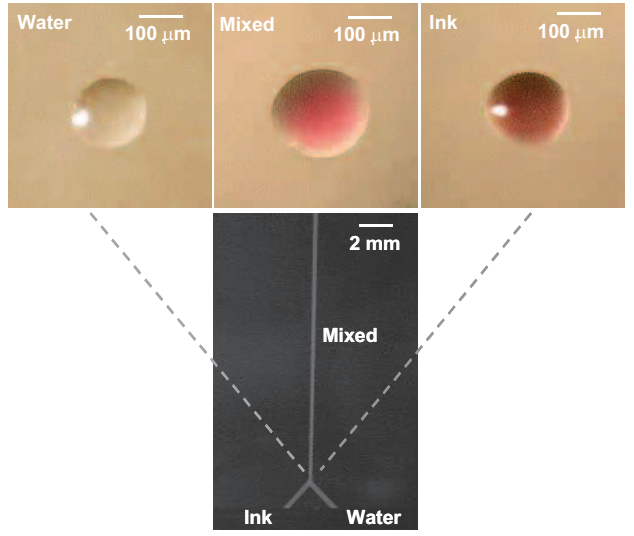

Figure 7. The water, ink, and mixed droplets ejected onto a glass slide by directional ejections. For comparison, one water droplet $(\sim 0.27 \mathrm{nl})$ was first ejected and collected, and then one ink droplet $(\sim 0.27 \mathrm{nl})$ was ejected and collected. Finally, one water and one ink droplet were simultaneously ejected and coalesced in air. The coalesced droplet continued traveling until it was collected.

\section{CONCLUSIONS}

We have demonstrated the controlled coalescence of liquid droplets in air and its applications to micromixing. Not only can the directional ejectors dispense nanoliter droplets of aqueous and non-aqueous fluids, it can also eject fluids with solid particles in various oblique angles for droplet coalescence and micromixing in air. This droplet dispensing method is capable of targeting a same spot in air with combinations of different reagents either concurrently or sequentially. A more flexible microfluidic system can be further constructed with arrays of multi-directional ejector having a set of electrically-tunable sector electrodes. Because of its high throughput, positional accuracy, and repeatability, directional acoustic ejection is suitable for many other applications, ranging from drug discovery to combinatorial chemistry.

\section{REFERENCES}

[1] H. Suzuki, C.-M. Ho, and N. Kasagi, "A Chaotic Mixer for Magnetic Bead-Based Micro Cell Sorter," Journal of Microelectromechanical Systems, 13, 779 (2004).

[2] C. Lee, H. Yu, and E.S. Kim, "Acoustic Ejector with Novel Lens Employing Air-Reflectors," IEEE MEMS Conference, Istanbul, Turkey, 1/22-26/06, pp. 170-173. 